\title{
Semantic facilitation in lexical decision as a function of prime-target association
}

\author{
ASHER KORIAT \\ University of Haifa, Haifa, Israel 31999
}

\begin{abstract}
Using a lexical decision task, the relationship between magnitude of semantic facilitation and degree of prime-target relatedness was examined as a function of amount of attention allocated to the prime and the prime-target interval. In none of the conditions studied did amount of facilitation vary with prime-target relatedness, a finding which was seen as inconsistent with the spread of activation account of the association effect in lexical decision. Both forward (prime to target) and backward (target to prime) associations were effective in producing semantic facilitation. Backward associates, however, were effective only during earlier stages of the experiment and forward associations only during later stages. The implications of these findings for the processes underlying the association effect was discussed.
\end{abstract}

A common assumption shared by several current theories of attention and memory (Collins \& Loftus, 1975; Posner \& Snyder, 1975a) is that the stimulation of a memory concept results in an automatic spread of activation to related concepts in the memory system. The spread of activation is assumed to follow the paths of the associative network and to fall off gradually as the spreading activation moves farther away from the original concept. The notion of a spreading activation has been used to explain a variety of phenomena, such as differences in the time required to verify semantic relations (Collins \& Loftus, 1975), the false recognition of related words in recognition memory tests (Underwood, 1965), the priming effect in the Stroop task (Warren, 1972) and the contextual facilitation effect in the lexical decision task (Schvaneveldt \& Meyer, 1973).

Another common assumption in current theories of information processing involves the distinction between attentional and automatic processes (Posner \& Snyder, 1975a; Schneider \& Shiffrin, 1977; Shiffrin \& Schneider, 1977). Automatic processes are assumed to operate without the subject's intention, without conscious awareness and without depleting the resources of a limited-capacity central processor. They are mostly involved in the utilization of habitual, overlearned associations. Attentional processes, on the other hand, operate on a conscious level, are under the control of the subject, and involve a limited-capacity mechanism.

This research was carried out while the author was a Visiting Associate Professor at the University of Oregon. It was supported by National Science Foundation Grant BNS 76-18907 to Michael Posner. I would like to thank Michael Posner for his invaluable help and support, and the members of the Center for Cognitive Studies for the use of their facilities. C. Osgood helped in the programming of the experiments. Requests for reprints should be addressed to Asher Koriat, Department of Psychology, University of Haifa, Haifa, Israel 31999.
Posner and Snyder (1975b) presented evidence indicating that the effects of a prime on the processing of a subsequent item vary depending on the amount of active attention committed to the prime. With low levels of attention, a prime tends to produce only facilitatory effects, whereas with high levels of attention, it produces facilitation as well as inhibition.

The present study originally intended to examine the manner in which the spread of activation from a priming concept to related concepts in an associative network might vary as a function of the amount of attention committed to the priming concept. Consider a priming concept, $P$, and three related test concepts, $T_{1}, T_{2}$, and $T_{3}$, arranged in terms of increasing semantic distance from $P$. The effects of the presentation of $P$ on the processing of $T_{1}, T_{2}$, and $T_{3}$ could be evaluated by comparing the processing of these test concepts when they are followed by a control, an unrelated concept. Since most theories assume that activation is attenuated the farther away it travels from its source, the facilitatory effects of the prime are expected to display a corresponding gradient from $T_{1}$ to $T_{3}$. The question of interest to the present research was whether and in what way the spreading activation gradient would vary depending on the amount of attention committed to the prime. Figure 1 depicts one possible hypothesis regarding the expected differences: The gradient with which the facilitatory effects of the prime fall off with increasing distance from the prime should be steeper with high attention to the prime than with low attention to the prime. The pattern displayed in Figure 1 depicts a particular interaction: Although the amount of facilitation is smaller on the average for low-attention than for highattention primes, for remotely related concepts $\left(T_{3}\right)$ a nonattended prime may have stronger effects than an attended prime.

This hypothesis was most explicitly stated by Spence and Holland (1962), but was formulated in terms of 


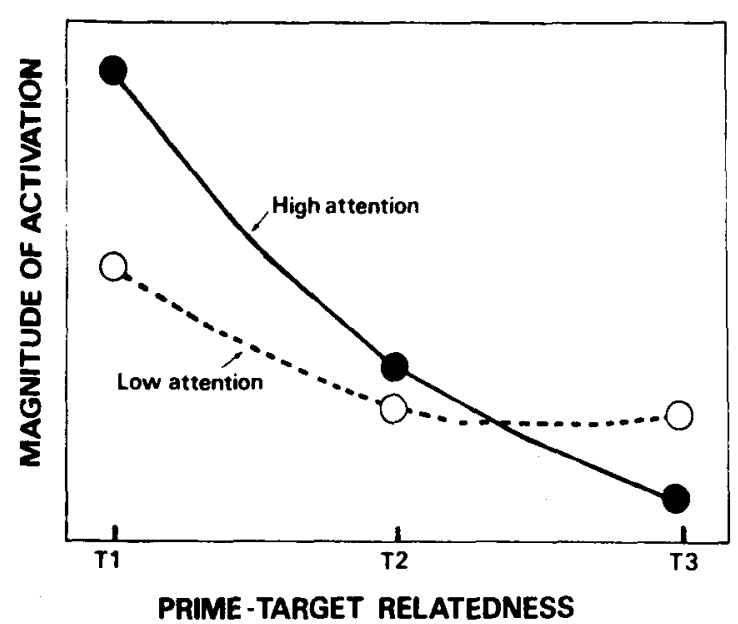

Figure 1. Predicted patterns of activation spread as a function of amount of attention committed to the prime and degree of prime-ts : : ratedness.

levels of awareness rather than in terms of levels of attention. Spence and Holland's (1962) thesis, derived from psychoanalytic theory, was that awareness of a stimulus has a restricting effect upon the range of related stimuli it can activate. With reduced awareness, more associative pathways become available and activation tends to fan out to a broader range of associates. Although Spence and Holland (1962) presented evidence that is consistent with this thesis, there is some doubt about the replicability of their original results (Barber \& Rushton, 1975; Spence \& Smith, 1977).

The hypothesis that active attention and awareness of a prime may have a restricting effect deserves a fresh examination in view of some recent work in the area of information processing. First, it is consistent with the two-process theory formulated by Posner and Snyder (1975a). Active attention to a prime should bring to bear the inhibitory processes characteristic of the limited-capacity mechanism, and these may be expected to impose a limitation on the number of concepts that can be activated. Second, Fischler and Goodman (1978) have recently reported an intriguing finding that is in line with the restricting-effects-of-awareness hypothesis. Subjects were exposed to a very brief presentation of word prime followed by a target stimulus to which a lexical decision had to be made. Although responses to the target were facilitated by a related prime, this effect was much larger for trials in which the prime was not identified than for trials in which the prime was identified.

The lexical decision task appears to be a most suitable method for examining patterns of spreading activation. Numerous studies indicated that when subjects have to decide whether a string of letters (e.g., "butter") is a word, decision latencies are shorter when the string of letters is preceded by a related priming word (e.g., "bread") than when it is preceded by an unrelated word (e.g., "nurse"). This effect has been customarily seen to reflect the automatic spread of activation from the prime word to related words (Fischler \& Goodman, 1978; Meyer \& Schvaneveldt, 1971). Accordingly, the design of the first experiment was as follows: On each trial a prime word serving as a warning signal was presented, followed by a stimulus to which a lexical decision task had to be made. The prime-target word pairs for the critical trials were selected on the basis of word association norms to represent three levels of associative relatedness. The method used by Posner and Snyder (1975b) was employed to create two levels of attention to the prime by manipulating the probabilities that the prime word was followed by a related word. In the highvalidity condition, $80 \%$ of the test words were preceded by a related prime, whereas in the low-validity condition, only $20 \%$ of the words were preceded by related primes. It was hoped that more attention or processing capacity would be committed to the prime when it served as a valid predictor of the subsequent test word.

Two previous studies (Becker, 1980; Schmidt, 1976b) examined hypotheses which bear some similarity to the hypothesis depicted in Figure 1. Schmidt (1976a) varied both intensity of context, defined in terms of the number of primes preceding a test word, and average degree of prime(s)-test relatedness. Although highly related contexts produced stronger effects than moderately related contexts, the expected pattern of a steeper relatedness gradient for high-intensity than for low-intensity contexts was not obtained. This result was seen by Schmidt to support an automatic activation explanation of the association effect.

Becker (1980) varied intensity of context by using lists with predominately strong, and lists with predominately weak, prime-test relationships. Only for the former lists did the magnitude of the association effect vary with the strength of the relationship within the individual pairs. If average associative relatedness can be assumed to achieve its effect by affecting level of attention to the prime, the results of Becker's study can be seen to be consistent with the hypothesis of the present study.

\section{EXPERIMENT 1}

\section{Method}

Subjects. Twenty University of Oregon students were recruited from the subject pool of the Center for Perceptual and Cognitive Studies and were paid $\$ 2.50$ for their participation. All subjects were native English speakers.

Procedure. Subjects were run individually in a darkened sound-attenuated room. They were told that they had to classify strings of letters as words or nonwords as quickly as they could, and that each string of letters would be preceded by a word that would serve as a warning signal. In the high-validity condition, subjects were also informed that, if the string of letters constituted a word, it would be related to the warning signal in $80 \%$ of the trials, and they were encouraged to take advantage of this relationship in responding to the test item. 
The experiment consisted of a series of 220 experimental trials, preceded by 22 practice trials. The experiment was controlled by an on-line PDP/15 computer. Each trial was initiated by presenting the prime word for $550 \mathrm{msec}$ at the center of a cathode-ray tube (CRT) display. No overt response was required to the prime. The prime was replaced after $100 \mathrm{msec}$ by the test word, which was presented so that its first letter occupied the same location as the first letter of the prime. Subjects classified the letter string as either a word or a nonword by pressing one key (labeled "word") with the right index finger for words and another key (labeled "nonword") with the left index finger for nonwords. The subject's response terminated the display of the test stimulus and initiated a $1-\mathrm{sec}$ intertrial interval.

Stimulus materials. Two hundred and twenty stimulus pairs, which included 60 critical pairs, 90 filler pairs, and 70 wordnonword pairs, were compiled. The 60 critical pairs were compiled from the word association norms of Palermo and Jenkins (1964) to form three sets of 20 pairs each, representing three levels of associative strength. Associative strength was determined by the probability of occurrence of the second word of a pair (target) as an associate of the first (prime) among college students. The average probabilities of associations for the lowmedium-, and high-associative strength sets were .065 (range .025 to .118 ), .257 (range .208 to .354 ), and .564 (range .408 to .706 ), respectively. The range and average frequency of occurrence in English of the response members in the three sets were approximately equal: Average frequencies of occurrence (Kucera \& Francis, 1967) for the low, medium, and high sets were 100,97 , and 105 , respectively. In choosing the pairs, a deliberate attempt was made to include a variety of word association types, such as coordinates, contrasts, predicates, and so on (see Moran, 1966), and the proportion of these types in the three sets were also approximately equal.

The 90 filler pairs were selected so that the range and average probability of association among college subjects were comparable to those of the 60 critical pairs. All 90 filler pairs were selected from Palermo and Jenkins (1964) except for 8 pairs that were selected from Keppel and Strand (1970).

For the nonword trials, a set of 140 words was compiled. Half of these were used to create the nonwords by randomly replacing one letter with a different letter of the same class to form a pronounceable nonword. The remaining words were used as primes in the nonword trials.

Design. A 2 by 3 by 2 (validity by associative strength by context) design was used, with validity varied between subjects and associative strength and context (related, unrelated) varied within subjects. The validity manipulation was created through variations in the filler items. In the high-validity condition, all the filler pairs were presented so that the stimulus member appeared as the prime and its response member in the word association norms appeared as the corresponding test word. For the low-validity condition, all the filler pairs were re-paired to form unrelated words.

Stimuli for the critical trials were generated as follows: All subjects received the response members of the 60 critical pairs as test words. For each subject, 30 of these, 10 of each associative strength set, were preceded by their corresponding stimulus words as primes, and the remaining 30 were preceded by an unrelated prime. The unrelated pairs were generated by randomly re-pairing each test word with any of the remaining 29 primes. Test words were assigned to related and unrelated primes in such a way that all test items appeared with both a related and an unrelated prime across each pair of successive subjects.

Thus, in the low-validity condition, only $30(20 \%)$ of the 150 test words appeared with a related prime, whereas in the highvalidity condition, $120(80 \%)$ of the test words appeared with a related prime.

The order of the experimental trials was such that each block of 22 successive trials included nine filler trials, one of each of the context by associative strength conditions. The two practice lists, used with the high-validity and the low-validity conditions, were constructed in a manner similar to that of the respective experimental blocks. Thus, for the high-validity group, 12 of the 15 test words were preceded by a related prime compared with 3 words for the low-validity group.

The order of the word-nonword pairs and of the filler test words was the same for all subjects, as were the ordinal positions of the critical trials. The assignment of the critical items to different ordinal positions was determined randomly for each subject.

\section{Results and Discussion}

Mean latency of correct responses on the critical trials was calculated for each of the validity by associative strength by context conditions. These are shown in Table 1. Two analyses of variance were carried out: The first involved averaging over items and using subjects as a random variable, and the second involved averaging over subjects and using items as a random variable. The $F$ ratios from these analyses are designated $F_{s}$ and $F_{i}$, respectively.

In both analyses, the effects of context were highly significant $\left[F_{s}(1,18)=32.41, p<.001 ; F_{i}(1,38)=19.82\right.$, $\mathrm{p}<.001]$. Overall, net facilitation due to a related prime was $31.5 \mathrm{msec}$. The subject-based analysis yielded a significant effect of associative strength $[F(2,36)=5.77$, $\mathrm{p}<.001\}$, apparently reflecting the fact that test words in the low-associative strength pairs took somewhat longer to classify than did words in the other two sets. The item-based analysis yielded a significant effect for validity $[F(1,38)=14.72, p<.001]$, reflecting the fact that the responses of the high-validity group were faster on the average by about $31 \mathrm{msec}$ than those of the lowvalidity group. In both analyses the Validity by Context interaction approached significance $\left[F_{s}(1,18)=3.52\right.$, $\mathrm{p}<.10 ; \mathrm{F}_{\mathrm{i}}(1,38)=2.27$, n.s.]. In neither analysis did the Context by Associative Strength interaction approach significance.

The results from the two analyses were combined to estimate $\min \mathrm{F}^{\prime}$ (Clark, 1973). The effect for context was highly significant $\left[\min F^{\prime}(1,55)=12.29, p<.001\right]$. No other effect was significant.

Mean response latency for the filler word-word trials was $496 \mathrm{msec}$ (1.7\% errors) for the high-validity group

Table 1

Mean Response Latency (RT) in Milliseconds and Percent Error (PE) for Unrelated and Related Prime Trials by Validity and Associative Strength in Experiment 1

\begin{tabular}{|c|c|c|c|c|c|c|}
\hline \multirow[b]{3}{*}{ Context } & \multicolumn{6}{|c|}{ Associative Strength } \\
\hline & \multicolumn{2}{|c|}{ Low } & \multicolumn{2}{|c|}{ Medium } & \multicolumn{2}{|c|}{ High } \\
\hline & RT & $\mathrm{PE}$ & RT & PE & RT & PE \\
\hline & \multicolumn{6}{|c|}{ Low Validity } \\
\hline Unrelated & 578 & 4 & 550 & 3 & 563 & 1 \\
\hline Related & 563 & 1 & 545 & 0 & 520 & 0 \\
\hline \multirow[t]{2}{*}{ Net Facilitation } & 15 & & 5 & & 43 & \\
\hline & \multicolumn{6}{|c|}{ High Validity } \\
\hline Unrelated & 555 & 1 & 532 & 4 & 540 & 2 \\
\hline Related & 506 & 1 & 496 & 1 & 500 & 1 \\
\hline Net Facilitation & 49 & & 36 & & 40 & \\
\hline
\end{tabular}


and $570 \mathrm{msec}$ ( $2.4 \%$ errors) for the low-validity group. These values are comparable to the mean latency for related critical trials $(500 \mathrm{msec})$ and unrelated critical trials $(563 \mathrm{msec})$ for the respective groups. Mean latency on the word-nonword trials was 617 for the high-validity (7.7\% errors) and 693 (4.4\% errors) for the low-validity group. The pattern of results for the word-nonword trials suggests that subjects in the high-validity group adopted a faster responding set than did subjects in the low-validity group.

The finding that degree of facilitation does not vary systematically with associative strength under either of the validity conditions might not be surprising in view of Fischler's (1977a, 1977b) results. He compiled a list of word pairs that were judged by him to be semantically related despite the fact that they had zero normative association (e.g., bread-cake). Using a lexical decision task, he obtained context effects for the pairs that were no weaker than those found for associatively related pairs. This finding indicates that there are dimensions of relatedness, pertinent to the context effect in lexical decisions, that are not captured by word association norms. What is more, for the associatively related pairs, magnitude of context effects was unrelated to normative associative strength.

These results, however, were obtained with simultaneous presentation of the word pairs. In a subsequent study (Fischler \& Goodman, 1978), in which successive presentation was used, magnitude of context effects was significantly correlated with associative strength.

The absence of an associative strength effect in the high-validity condition of the present study is particularly surprising in view of previous work on the effects of expectancy on the activation of semantic memory. This work suggests that expectations for a particular set of elements tend to produce a bias in favor of the more representative or typical elements in this set. Loftus (1973) asked subjects to decide whether a word was a member of a verbal category. When the category name preceded the word, response latency was shorter for more representative instances than for less representative instances of the category, as measured by the normative frequency with which the instances were given as members of that category. Similarly, Rosch (1975a), using same-different judgments, found that advance priming with the category name facilitated responses to good examples and hindered responses to poor examples for physically identical pairs. Here, too, the selective effect of the prime was entirely eliminated by a simultaneous presentation of prime and stimuli.

The high-validity condition of the present study bears obvious similarities to the conditions investigated by Loftus and by Rosch, and it is therefore puzzling that no differential effect of the prime on high and low associates was revealed. Perhaps the prime onset/target onset interval employed in the present experiment was too short to allow generation of articulate expectations. Rosch (1975a, 1975b), for example, found that when the interval between the termination of the auditory prime and the onset of the visual stimuli was reduced below $500 \mathrm{msec}$ the interaction between priming and goodness of example was greatly diminished. Accordingly, in Experiment 2 a longer prime-target interval was employed. It was expected that, in the high-validity condition, the longer interval would allow the generation of more differentiated expectations which would result in a stronger facilitation for high than for low associates of the prime.

\section{EXPERIMENT 2}

\section{Method}

Subjects. Twenty new subjects recruited from the same source as that in Experiment 1 were paid for their participation.

Stimuli and Procedure. The stimuli and procedure were identical to those of Experiment 1 except that the prime was presented for $1,500 \mathrm{msec}$ and was replaced after $100 \mathrm{msec}$ by the test.

\section{Results and Discussion}

Table 2 presents mean reaction times for correct responses and percent errors on the critical trials for the validity by associative strength by context conditions.

Two analyses of variance, one using subjects and one using items as the random variable were carried out. In both analyses the Context by Validity interaction was significant $\left[F_{s}(1,18)=41.80, p<.001\right.$; $\left.F_{i}(1,38)=13.25, p<.001\right]$, as were the main effects of context $\left[F_{s}(1,18)=20.15, p<.001 ; F_{i}(1,38)=7.57\right.$, $p<.01]$. It may be seen from Table 2 that the effects of context are substantial in the high-validity condition but are entirely absent in the low-validity condition. The effects of validity were significant only in the item-based analysis $\left[F_{i}(1,38)=5.35, p<.05\right]$. Associative strength yielded significant main effects $\left[F_{s}(2,36)=9.73, p<.001 ; F_{i}(2,76)=9.78, p<.001\right]$, reflecting the fact that the low associative strength test words took somewhat longer to classify than did words in the other two sets. However, none of the interactions involving associative strength was significant.

Table 2

Mean Response Latency (RT) in Milliseconds and Percent Error (PE) for Unrelated and Related Prime Trials by Validity and Associative Strength in Experiment 2

\begin{tabular}{|c|c|c|c|c|c|c|}
\hline \multirow[b]{3}{*}{ Context } & \multicolumn{6}{|c|}{ Associative Strength } \\
\hline & \multicolumn{2}{|c|}{ Low } & \multicolumn{2}{|c|}{ Medium } & \multicolumn{2}{|c|}{ High } \\
\hline & RT & PE & RT & $\mathrm{PE}$ & RT & PE \\
\hline & \multicolumn{6}{|c|}{ Low Validity } \\
\hline $\begin{array}{l}\text { Unrelated } \\
\text { Related } \\
\text { Net Facilitation }\end{array}$ & $\begin{array}{r}577 \\
577 \\
0\end{array}$ & $\begin{array}{l}5 \\
2\end{array}$ & $\begin{array}{l}533 \\
562 \\
-29\end{array}$ & $\begin{array}{l}1 \\
3\end{array}$ & $\begin{array}{r}546 \\
546 \\
0\end{array}$ & $\begin{array}{l}3 \\
1\end{array}$ \\
\hline & \multicolumn{6}{|c|}{ High Validity } \\
\hline $\begin{array}{l}\text { Unrelated } \\
\text { Related } \\
\text { Net Facilitation }\end{array}$ & $\begin{array}{r}632 \\
579 \\
53\end{array}$ & $\begin{array}{l}5 \\
1\end{array}$ & $\begin{array}{r}577 \\
545 \\
32\end{array}$ & $\begin{array}{l}1 \\
0\end{array}$ & $\begin{array}{r}595 \\
520 \\
75\end{array}$ & $\begin{array}{l}3 \\
0\end{array}$ \\
\hline
\end{tabular}


Error rate for unrelated trials was $3 \%$ for both of the validity conditions. For related trials, however, error rate was $2 \%$ for the low-validity condition compared with $.3 \%$ for the high-validity condition.

Overall, it can be seen in comparing the results of Experiments 1 and 2 that the effects of the validity manipulation on the extent of facilitation are greater with longer prime-target interval. Nevertheless, even in Experiment 2, in which validity had a clear effect on the extent of facilitation, the effects of the prime in the high-validity condition were not significantly different for the three levels of associative strength employed.

Considering only the high-validity conditions of Experiments 1 and 2, the results indicate, first, that the processing of the stimulus words was facilitated by the presentation of related primes and, second, that the magnitude of the facilitation effect was unrelated to the degree of relationship between the prime and the word target.

These results appear to raise some serious difficulties for the commonly accepted account of the associative effect in lexical decisions in terms of the "spread of activation" theory (Collins \& Loftus, 1975). According to this theory the retrieval of information from one memory location produces automatic spread of activation to other nearby locations, facilitating the subsequent access to information stored there. The effect of a prime on the lexical decision response to a related word conforms to this theory; the finding that the size of this effect was indifferent to the strength of the prime-target associative link, however, does not.

The assumption that a concept primes a closely related concept to a greater extent than it primes a less closely related concept has been used by Loftus (1975) to explain the priming results of Rosch $(19751,1975 \mathrm{~b})$ in terms of the spreading activation theory. Assuming that the production frequency of an instance to a category name reflects the strength or accessibility of the category name/instance name connecting path, it follows that a category name should facilitate responses to good examples to a greater extent than should responses to poor examples, as was indeed found. The result that a category name actually hindered responses to poor examples was interpreted by Loftus (1975) in terms of the additional assumption that, if the total amount of activation is limited, the priming of one concept by a closely related concept may make a third, distant concept temporarily less accessible.

The discrepancy between the pattern of results obtained in the present study and that reported by Rosch (1975a, 1975b) raises doubts about the assumption that both, the priming effect in Rosch's categorization task and the association effect in lexical decision, reflect one and the same underlying process-spread of activation. It may still be argued, however, that the measures of word relatedness employed in the two studies are not equivalent, one being based on word association norms, the other on categorization norms.
There is yet a third effect with regard to which the concept of spreading activation has been invoked. It involves the Stroop effect reported by Warren (1972). Warren presented an auditory item followed by a visual word printed in colored ink and found that the time for naming the ink color was longer when the visual word and the prime were associatively related than when they were not. This effect was interpreted as reflecting the activation of the word by the related prime, which increases its interference with saying the word (Posner \& Snyder, 1975a). In a subsequent study (Warren, 1974), the strength of the associative relationship between the auditory prime and the visual word (as measured by word association norms) was found to have a significant effect on the extent of interference observed. Thus, for word pairs with low associative strength there was a 20 -msec interference, compared with $95 \mathrm{msec}$ for the high associative strength pairs. It should be pointed out that the range of associative strengths sampled by Warren was comparable to that used in the present study (the mean associative strength for the low and high groups were $15.2 \%$ and $63.0 \%$, respectively, in Warren's study and $6.5 \%$ and $56.4 \%$, respectively, in the present study).

If both the Warren effect and the association effect in lexical decisions are assumed to reflect the same underlying process, the discrepancy between the results of the present study and those of Warren (1974) is disturbing, particularly in view of the fact that in both studies semantic distance was defined in terms of the same measure. If, however, the two phenomena reflect different processes, one may expect additional systematic differences between them. Experiment 3 was intended to explore this possibility.

\section{EXPERIMENT 3}

One assumption that is basic to the spreading activation metaphor is that the flow of activation is directional. Activation is said to spread from the source outward, from the prime concept to the target concept. The amount of activation gained by a target concept as a result of the processing of a prime should therefore be a function of the strength of the "forward" links from the prime to the target and should be independent of the strength of the "backward" links from the target to the prime.

The results obtained in a categorization task have been shown by Loftus (1973) to conform to this assumption. Subjects had to decide whether or not a word was a member of a category. When the category preceded the word, reaction time was related to the frequency with which the word was evoked by the category name and unrelated to the frequency with which the word evoked the category. When the word preceded the category, on the other hand, the reverse pattern was found.

A similar finding was reported by Warren (1974) 
with the Stroop task. Using unidirectional associates he found that only the association in the forward direction (from the prime to the color word) increased interference of color naming; backward association had no effect at all.

These results are consistent with the hypothesis that the priming effect in both the categorization task and the Stroop task are due to a spreading activation mechanism. If this mechanism is assumed to be responsible for the association effect in lexical decision as well, we should expect the occurrence of the association effect to depend on forward associations.

Somewhat different predictions follow from another explanation of the association effect based on the location shift hypothesis. According to this hypothesis only one memory location can be read out at a time, and the time to shift from one location to another increases with the distance between the two locations. Thus, the association effect is assumed to occur because the shifting to nearby locations is faster than shifting to more distant locations. Studies contrasting the location shift hypothesis with the spreading activation hypothesis yield evidence which appears to favor the former (Schvaneveldt \& Meyer, 1973).

In the absence of additional assumptions regarding the location shift model, it would seem plausible to assume that the concept of "distance" pertinent to this model is a symmetrical one: The time to shift between two locations should be the same whichever location is used as a starting point. Thus, the extent of facilitation of one word by another should be unaffected by the order in which the words are processed. One possible measure of distance which might be used in connection with the location shift hypothesis is the Mutual Relatedness Index (Jenkins \& Cofer, 1957), which takes into account the extent to which two words tend to evoke each other as well as common associates.

There are several observations that point in the direction of still a third model, one which emphasizes the importance of backward associations. In the spread of activation explanation, as commonly described, the automatic activation of a particular memory entry through priming is assumed to take place prior to the encoding of the word corresponding to this entry, regardless of whether or not the word is presented. In the proposed "double take" hypothesis, the association effect is assumed to take place following the complete or partial encoding of the test word. Once the test word has been processed, it tends to "reactivate" or reinstate the related prime. The lexical decision task is then carried out "in the context" of the trace of the related prime, a condition which, for an as yet unknown reason, facilitates decision.

There are several observations that support the possibility of such a process. First, according to subjects' introspections, awareness of the prime was stronger when it was followed by a related word than when it was followed by an unrelated word. Second, Fischler and Goodman (1978) observed that in a lexical decision task with a very short prime-test interval, the prime was correctly recalled more often when followed by a related word than when followed by an unrelated word. A similar finding was reported by Jacobson (1973): Words were more readily reported when masked by their associates than when masked by unrelated words.

Thus, if this proposition is correct, it follows that the occurrence of the association effect will depend on the existence of a backward association from the test word to the prime. The size of the association effect may not depend in this case on the strength of the association, for reasons which will be discussed later.

In Experiment 3 pairs of words with unidirectional associations were employed. The order of the members of each pair was systematically varied so that the association was in a forward or in a backward direction. The results should have implications regarding the three accounts of the association effect outlined above. An association effect in the forward direction alone supports the spread of activation explanation, an effect in the backward direction alone supports the "double take" explanation, and an effect that is independent of the direction of association will conform to the location shifting hypothesis.

\section{Method}

Subjects. Twenty-four subjects participated in Experiment 3. All were recruited from the same pool as in Experiment 1 and paid for their participation, except for eight subjects who participated for course credit.

Procedure. The apparatus was identical to that used in the previous experiments. Subjects were instructed to classify the visually presented letter string by pressing the left-hand key for nonwords and the right-hand key for words. In each trial a prime word served as a warning signal and appeared for $550 \mathrm{msec}$. It was replaced after $100 \mathrm{msec}$ by a test letter string. The subject's response terminated the display and initiated a 1 -sec intertrial interval. As in the low-validity condition of the previous experiments, subjects were informed that the warning signal was always a word, but no mention was made of its possible relationship to the letter string.

The experiment included two presentations of a series of 192 trials. The first presentation was preceded by 28 practice trials. A short break occurred between the two presentations. Each presentation included four blocks of 48 trials each. At the conclusion of the practice block and of each of the experimental blocks, a feedback display appeared on the CRT which indicated the average reaction time and the number of errors on that block. The significance of these values was explained at the end of the practice trials, and subjects were instructed to try to improve their performance with successive blocks. Within each presentation subjects were allowed to proceed to the next block whenever they felt ready.

Design and Materials. The stimuli for the practice block included 7 associated word-word pairs, 7 unrelated word-word pairs, and 14 word-nonword pairs. The associated word-word pairs were selected from Keppel and Strand (1970) so that for each pair the forward and backward associations were approximately of equal probability of occurrence.

The stimuli for the word-word pairs of the experimental blocks were those used by Warren (1974), with a slight modification. In Warren's study 12 word pairs were presented with the association in a forward direction and 12 pairs were presented with the association in a backward direction. In the present study the 
direction of association was manipulated within pairs, each of the 24 pairs appearing both in the forward and in the backward direction. This modification required the use of two control words (rather than one), in connection with each pair of associated words, to serve as primes in the unrelated conditions. Thus, there was a total of 24 word quadruples out of which all the stimuli for the critical trials were formed.

In addition, 192 words were compiled to form 96 wordnonword pairs. The nonwords were generated by randomly replacing one letter with another letter of the same class to form a pronounceable nonword.

Each of the four experimental blocks included 48 trials, 24 involving word-nonword pairs, 12 involving associated wordword pairs, and 12 involving unrelated word pairs. The first trial in each block involved a word-nonword pair, but apart from this the position of the word-nonword pairs was random and was the same for all subjects.

The construction of the word-word pairs was as follows. Let the four words of each quadruple be designated $A, B, C$, and D, with $A$ and $B$ representing the associated words so that the direction of the (dominant) association is from $A$ to $B$ and with $C$ and $D$ representing the control words. Four different prime-test patterns could be formed (the first letter designating the prime word): A-B (forward), B-A (backward), C-A (control backward), and $D-B$ (control forward).

The following procedure was employed to assign each word quadruple to each of the four patterns. For each of the blocks, exactly six quadruples appeared in each of the four patterns (each quadruple contributing only one pattern), and for each subject each quadruple appeared in all four patterns across the four blocks. The same assignment schedule was employed for each group of four successive subjects, except that the order of administration was rotated so that the stimuli used for the second block of the first subject were used for the first block of the second subject, and so on. In this manner, considering only the first block of each subject, all 48 test words appeared with both a related and an unrelated prime across each group of four successive subjects. The order of the various word-word pairs in each block was otherwise random.

Over all 24 associated pairs the average associative strength from $A$ to $B$ was .40 (range $.225-.723$ ) compared with .02 for the association from $B$ to $A$. The 24 quadruples were divided at the median of the $A$ to $B$ association to form two equal sets. The mean probability of the $A$ to $B$ association in the high associative strength set was .522 compared with .027 for the B to A association. The respective figures for the low associative strength set were .272 and .013 . After the first 12 subjects of Experiment 3 were tested an additional restriction was imposed on the assignment of quadruples to patterns: Half of the A-B and half of the B-A pairs had to be from the high associative strength sets and the remaining halves from the low associative strength sets.

\section{Results and Discussion}

Table 3 presents percentage of errors and mean response latencies for correct responses on the critical trials for each of the direction by context by presentation conditions. A direction by context by presentation analysis of variance with subjects as the unit of analysis yielded significant main effects for presentation $[F(1,23)=36.08, p<.001]$, for context $[F(1,23)=$ $33.076, p<.001]$, and for direction $[F(1,23)=19.702$, $p<.001]$. None of the two-way or three-way interactions was significant. The same pattern of results was obtained in an analysis of variance using items as the unit of analysis, except that the Presentation by Context interaction was also significant.
Table 3

Mean Response Latency (RT) in Milliseconds and Percent

Error (PE) for Unrelated and Related Prime Trials by Direction and Presentation

\begin{tabular}{lcccc}
\hline & \multicolumn{4}{c}{ Direction } \\
\cline { 2 - 3 } \multicolumn{1}{c}{ Context } & \multicolumn{2}{c}{ Forward } & \multicolumn{2}{c}{ Backward } \\
\cline { 2 - 3 } \cline { 5 - 5 } & RT & PE & RT & PE \\
\hline Unrelated & \multicolumn{4}{c}{ Presentation 1 } \\
Related & 571.15 & 4.0 & 600.91 & 7.8 \\
Net Facilitation & 545.53 & 4.5 & 573.23 & 5.0 \\
& 25.62 & & 27.68 & \\
Unrelated & & Presentation 2 & \\
Related & 529.51 & 4.3 & 542.19 & 6.1 \\
Net Facilitation & 487.60 & 3.1 & 499.41 & 3.7 \\
\hline
\end{tabular}

Examination of Table 3 indicates that response latencies are shorter for the second than for the first presentation and for related than for unrelated contexts. The significant main effect of direction reflects a systematic between-word difference. Suppose all related word pairs were arranged in a forward direction (e.g., kittens-cats); the latencies to the first words were generally longer than to the second words, when these were used as targets. This was true in both related and unrelated contexts. This difference may reflect a general principle regarding word associations which deserves further investigation. For the purpose of the present paper, however, this difference will be treated as a sampling error.

It is interesting to note that the effect of context appears to increase from the first to the second presentation, whereas that of direction appears to decrease. Only the former interaction, however, is significant in an item-based analysis of variance.

The absence of a Context by Direction interaction is clearly evident in the data in Table 3: For both presentations the size of the association effect is practically identical for the forward and backward directions.

The finding that in lexical decision priming in the backward direction is as strong as that in the forward direction stands in sharp contrast with the results reported by Warren (1974) for the Stroop task. There, priming was entirely confined to the forward arrangement. This discrepancy suggests that the association effect in lexical decision involves a process different from that underlying the priming effect observed with the color naming task. Thus, the results of Experiments 2 and 3, taken together, indicate that the association effect in lexical decision, unlike that of the Stroop task, is not affected by either the strength or the direction of the primetarget association.

What then is the mechanism underlying the association effect in lexical decision? The finding that the association effect is indifferent to the direction of the association may be interpreted as indicating that the 
underlying mechanism itself is inherently symmetrical or directionless. As mentioned above, the location shift explanation may be conceptualized as implying a mechanism in which the pertinent concept of distance is symmetrical. The observed symmetrical effect, however, may also represent a joint effect of two directional processes that operate in opposite directions and result in a seemingly directionless net effect.

This latter possibility deserves attention in view of the current distinction between two types of cognitive processes. Consider the possibility that the association effect has two components, an attentional component and an automatic-activation component (Posner \& Snyder, 1975a). The attentional component involves the intentional anticipation of the target word: A subject who has encountered a large number of related pairs develops a tendency to prepare to process a target word that is related to the prime. The anticipation of related targets, I propose, rests heavily on the presence of forward association, that is, associations that allow the prediction of the target on the basis of the prime.

The second, automatic component does not depend on intentional preparation. Fischler (1977a), for instance, has shown that associative facilitation may be obtained without expectancy. I propose that this automatic process leans primarily on the presence of backward target-to-prime associations. When a prime is followed unexpectedly by a related target, it tends to be reactivated by the target. The activated prime then facilitates the processing of the related target.

If these propositions are correct, we should expect that in the earlier trials the association effect will be determined primarily by the presence of backward associations. Forward associations, on the other hand, will become effective in later trials.

To examine this possibility, the results of Presentation 1 were analyzed by blocks. It should be recalled that, for each of the four blocks, each of the critical words appeared equally often in each of the four patterns across all subjects. A block by context by direction analysis of variance yielded a significant triple interaction $[F(3,69)=2.92, p<.05]$. Figure 2 presents the mean net facilitation (mean latency for unassociated minus mean latency for associated pairs) for each of the blocks for the forward and the backward orders. The interaction displayed clearly conforms to predictions. On the first block, backward associations produced marked facilitation, whereas forward associations seemed to have little effect. With increased practice the pattern reverses itself, so that by the fourth block practically all of the facilitation is due to forward associations.

The increase in the amount of forward facilitation with practice is consistent with the proposition that this facilitation is due to a process of intentional preparation. The observation that facilitation due to backward associations diminishes with practice, however, appears inconsistent with the proposition that backward effects are automatic. More research is needed to clarify the

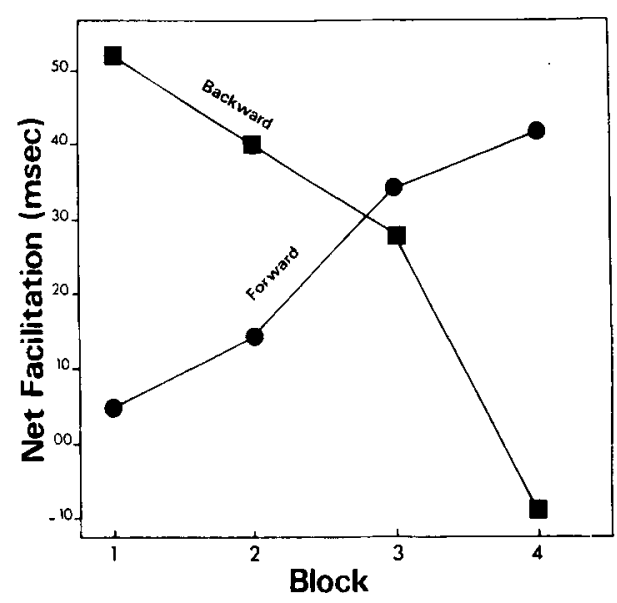

Figure 2. Mean net facilitation for forward and backward order for each of the experimental blocks.

precise pattern of development of backward and forward effects with practice before definite conclusions regarding the possible interactions between the two components of the association effect can be made.

Finally, several analyses were carried out to examine the possibility that the effects of associative strength on magnitude of facilitation will be different for the backward and the forward conditions. These analyses yielded no effects for associative strength under either condition during early or late stages of the experiment.

\section{GENERAL DISCUSSION}

The initial motivation for this program of research was to examine the idea that the pattern of activation spread from a prime varies as a function of the amount of attention allotted to it. The results, however, raised doubts regarding the appropriateness of the lexical decision task as a tool for testing this idea, and turned out to provide information regarding the lexical decision task itself.

In Experiment 1, using a prime duration of $550 \mathrm{msec}$, we found that degree of prime-target relatedness, as measured by word association norms, had little effect on the amount of facilitation. This was true for the two conditions studied, representing two levels of attention to the prime.

Experiment 2 examined the possibility that the expected effect of prime-target association on amount of facilitation might still be revealed if sufficient time were allowed for the generation of articulate expectations. This was assumed to result in a better preparation for high associates than for low associates of the prime. Use of a prime duration of $1,500 \mathrm{msec}$, however, did not result in any noticeable effect of association strength on magnitude of facilitation.

In Experiment 3 it was found that, on the average, backward associations were as effective as forward associations in facilitating responses to the target word. 
Moreover, there was an interaction with practice: Backward associations were most effective on the earlier trials, whereas forward associations were effective only in later trials.

The results on the whole cast some doubts on the assumption that a single mechanism underlies the context effects found in such tasks as the Stroop, the categorization task, and the lexical decision task (Collins \& Loftus, 1975).

The association effect may be seen to result from either or both of the following processes: a spreading activation process whereby the processing of a semantic element automatically activates all associated elements in the semantic network, or an active-attentional process involving the anticipation of, and the preparation for, a prime-related target word.

The spreading activation notion has found widespread use in a variety of conceptions, without an explicit theory regarding the details of the process. For the purpose of the following discussion we will refer to a particular version of the spreading activation model which assumes the following: first, that the spread of activation from a stimulated memory concept (prime) to related concepts (target) is automatic; second, that the extent to which a target concept is activated is proportional to its degree of association to the prime; third, that the spread of excitation is directional (that is, it proceeds along the associative paths from the prime to the target).

As far as the automaticity assumption is concerned, Fischler (1977a) provided evidence indicating that the association effect in the lexical decision task is automatic in the sense that its occurrence does not depend on the expectation of, and the preparation for, a prime-related word. Yet, results reported by Tweedy, Lapinsky, and Schvaneveldt (1977), as well as the results of Experiment 2 of the present study, indicated that the amount of associative facilitation varies with the proportion of related word pairs in the list. This finding suggests that, even if anticipation of a target is not a necessary condition for associative facilitation to occur, its presence enhances facilitation. This suggestion is in line with the notion (Posner \& Snyder, 1975a; Tweedy et al., 1977) that the association effect has both automatic and adaptive-attentional components.

Schmidt (1976b) also provided data supporting the two-process theory of context effect. Each trial in a lexical decision task was preceded by an uninformative cue or by a valid cue indicating that the test stimulus (when it was a word) was related or unrelated to the prime. Although the association effect was obtained in the absence of informative cuing, the presence of a cue was found to have a beneficial effect on the processing of both related and unrelated test words.

As far as the effects of prime-target relatedness is concerned, Collins and Loftus (1975) explicitly postulated that the amount of activation that spreads from a source along a given path is proportional to the strength of that path. They relied on this assumption in attempting to explain a variety of phenomena. As already noted, this relationship was demonstrated in connection with the Stroop task, which, according to Posner and Snyder (1975a), reflects an automatic activation process.

It should be pointed out, however, that the relationship between the amount of facilitation and the strength of the prime-target association would also be expected to hold if the lexical decision task is due to a selective preparation to process prime-related words. In this case, however, one would predict that the facilitation of expected stimuli would be coupled with the inhibition of unexpected stimuli (Posner \& Snyder, 1975a, 1975b). Indeed, several studies with the lexical decision task (Becker, 1980; Neely, 1976; Schubert \& Eimas, 1977) found that relative to a neutral context, a semantic context both facilitated the processing of related words and interfered with the processing of unrelated words. Rosch (1975a) found that priming with a category name facilitated responses to good instances of the category and actually hindered responses to poor instances for physically identical pairs of stimuli.

Experiments 1 and 2 of the present study failed to yield any evidence for the expected effects of primetarget relatedness on amount of facilitation. These effects were found neither for the low-validity condition, assumed to reflect automatic activation, nor for the high-validity condition, assumed to reveal the attentional expectancy-based process. The results remained unchanged even when a relatively long interstimulus interval was employed.

Previous research examining the effects of associative strength yielded inconsistent results. Thus, Fischler and Goodman (1978), using a lexical decision task, reported stronger facilitation for items with higher prime-test associative probability. On the other hand, Fischler (1977b), using simultaneous presentation, reported similar magnitudes of facilitation for associatively related pairs (e.g., dog-cat) and for semantically related pairs (e.g., nurse-wife) with zero normative association. For the associatively related pairs, amount of facilitation was, if anything, negatively correlated with associative strength.

Using a lexical decision task, Schmidt (1976b) varied prime-test relatedness by selecting word pairs that were both either instances of a narrow superordinate category (e.g., alcoholic beverages) or of a broader one (e.g., beverages). The magnitude of facilitation was significantly higher for the former than for the latter pairs.

Several results presented by Schmidt (1976a) suggested that the effects of prime-test relatedness on the magnitude of facilitation might be stronger the poorer the legibility of the test stimuli. A similar trend is evident in the data reported by Massaro, Jones, Lipscomb, and Scholz (1978): Prior priming with a category name facilitated naming and lexical decision when the test stimulus was rotated but not when it was normal. For 
rotated test stimuli, response to good exemplars was facilitated to a larger extent than response to poor exemplars.

Becker's (1980) work presents what is perhaps the best clue to the conditions under which associative strength effects are obtained. Using a lexical decision task, he found that lists containing strongly related prime-word pairs produced a context effect that was almost exclusively facilitatory. On the other hand, lists containing weaker relationships, resulted in little facilitation in the processing of prime-related targets and substantial interference in the processing of prime-unrelated targets. For the low-relatedness lists the magnitude of facilitation for a given prime-target pair was unrelated to the associative strength of the pair, whereas for the highrelatedness lists strongly related pairs exhibited stronger facilitation than did weakly related pairs.

In terms of the main hypothesis of the present research (see Figure 1), Becker's (1980) results are puzzling in that the shallow gradient of spread of activation was found precisely for the low-relatedness condition. In this condition the effects of the prime were primarily inhibitory. In fact, it appears as if relatedness operated as an all-or-none phenomenon in the lowrelatedness condition of Becker's study as well as in the conditions employed in the present study. In these conditions "related" pairs yielded different results from "unrelated" pairs, with degree of relatedness within the related set being inconsequential. I shall return to this phenomenon shortly.

Consider next the directionality of context effects. The spread of activation theory assumes that the flow of excitation is directional (Collins \& Loftus, 1975), and Warren (1974) has indeed demonstrated a significant effect of forward associations but not of backward associations in the Stroop task. However, as is the case with the associative strength effect, the directionality effect would also be expected even if the influence of context is seen to reflect an intentional, expectancy. based, rather than an automatic, process. This is because the critical factor in this case would be the extent to which the target word can be predicted on the basis of the prime, backward prediction being immaterial. Thus, directional effects are not necessarily a mark of automatic processes. This point is well illustrated by the dispute between Rosch (1975b) and Loftus (1975) regarding the interpretation of some of Rosch's findings.

In the present study no difference was found between forward and backward associations, a finding that is inconsistent with both views of context effects. The interaction with practice, however, suggested the operation of two distinct processes. Most intriguing is the finding that, during the earlier stages of the experiment, context effects were entirely confined to backward associations. A somewhat similar phenomenon has been observed by Razran (1949) in his studies on the semantic generalization of conditional responses, and was interpreted by him as suggesting that semantic general- ization is due to a process that takes place during the testing rather than during the learning stage.

It was proposed that as far as the lexical decision task is concerned, backward effects reflect an automatic process, whereas forward effects reflect primarily an attentional one. This interpretation might seem odd to proponents of the spreading activation model, since it is exactly the forward effects which should be attributed to automatic activation.

The proposition that the automatic component of context effects rests on backward associations has two important implications. The first pertains to the nature of the process involved; the second relates to the nature the interword associations that are critical for context effects.

Consider first the nature of the process involved. The proposition advanced above implies that the automatic context effect in lexical decision takes place after, not prior, to the presentation and partial encoding of the test word: The prime affects late stages of the encoding of the test word and/or the testing stage. It appears that a prime does not automatically affect the processing of a subsequently presented related word. It does so under two conditions: first, when the prime generates wellarticulated target-compatible expectations that are still active when the test word is presented; second, in the absence of such expectations, when the preliminary processing of the test word reactivates or reinstates the related prime. This second condition may be equivalent to presenting the prime and test word simultaneously or with a very brief interstimulus interval. When articulate expectations exist prior to the presentation of the target word, they may affect earlier stages of the encoding process as well. In the absence of such expectations, context effects will be confined to the testing stage and possibly to the completion of the encoding stage.

On the face of it, the effects of context under back. ward conditions appear rather puzzling. How can the word "cats" facilitate the processing of the word "kittens" if "cats" itself has to be activated by the word "kittens" to have an effect? Apparently, one of two assumptions is needed to explain this effect. The first assumes that the lexical decision task requires an additional operation over and above those needed for the encoding of the target word, and it is this operation that is affected by context. The second assumes that the process by which a prime is reactivated by a target word starts before the completion of the encoding of the target word and runs parallel to it.

The second implication involves the nature of the associative relations that are critical for the association effect to occur. The account presented above for context effects under backward conditions implies that the prime-test relationship serves two functions: First, it increases the likelihood that the completion of the encoding process and the testing process will be carried out "in the presence" of the prime. Second, it facilitates the processing of the test word. A similar distinction 
may be made with regard to context effect under conditions of forward associations, except that here the generation of test-appropriate expectations and the possible effect on early encoding stages must be taken into account. The relationship between these two functions of word relationships is not clear, since little is known about the mechanism by which one word affects the processing of a related word. Whatever this mechanism might be, however, it may be argued that the type of relationships that are pertinent to the testing stage (and probably to the late encoding stage) are different from those that are pertinent to the early encoding stage. We may propose a distinction between two indices of primetest relationships. The first, a priori relatedness, is pertinent to the encoding stage. It refers to the degree to which the test word can be predicted on the basis of the prime word. Normally, this type of relatedness is best captured by word association norms, which reflect the likelihood that the test word will be chosen from all other possible responses. The second, a posteriori relatedness, is pertinent to the late encoding and testing stages. It refers to the judged intensive relationship between the prime and the test when both are known. The critical difference between the two types of relatedness is that the nature of the other associates of the prime is crucial in determining degree of a priori relatedness but is entirely immaterial in determining a posteriori relatedness. To illustrate this point, let the reader guess the relative probability with which the second term in each of the following pairs is given as a response to the first term in word association norms: lamp-light, find-seek, sell-buy, beautiful-nice. The probability of association for the four pairs, respectively, are $.706, .025, .564$, and .028 (Palermo \& Jenkins, 1964). Most people who were asked to guess these probabilities underestimated greatly the differences among the pairs. Apparently, when both the stimulus and response terms are presented there is a tendency to ignore the role of other possible responses to the stimulus word. This effect might be analogous to the tendency to disregard base-rate information in probability judg. ments (Ajzen, 1977).

These considerations may help relate the results of Experiments 1 and 2 to those of Experiment 3. Given that the association between the prime and the test is sufficiently strong to establish a connection between them during the processing of the test word, the extent of the context effect will depend on a posteriori relatedness rather than on a priori relatedness, and therefore degree of facilitation would not necessarily be related to probability of association as reflected in word association norms. This proposition is consistent with the finding of Fischler (1977b) that nonassociated words such as "nurse-wife" may still yield a facilitation effect. This effect, according to the present formulation is due to their a posteriori relationship, that is, to the relation- ships discovered or realized when both are presented together. If this proposition is correct, it may explain why under some conditions relatedness between prime and test words seems to operate as an all-or-none phenomenon.

The probability of backward associations requires great care in the interpretation of results based on several experimental paradigms using priming effects. Two examples will suffice to illustrate this point. One experimental paradigm used to study the processing of lexically ambiguous words involves presenting a homograph in a context that biases interpretation toward one meaning and measuring its effect on the processing of test words related to the biased-for or biased-against meaning of the homograph (Schvaneveldt, Meyer, \& Becker, 1976; Hekannen, Note 1; Killion, Note 2; Swinney, Note 3). Results cited in support of the contention that both meanings are accessed must be carefully examined to assure that the effects of the biased-against meaning are not due to a backward activation process in which the test word reactivates the biased-against meaning. Since this backward activation takes place after, not before, the presentation of the test word, it should not imply that both meanings of a homograph are normally accessed. Such a backward activation, however, may explain the disambiguation of a homograph by a subsequent context.

The second example involves the paradigm employed by Schvaneveldt and Meyer (1973). They found that the magnitude of the association effect was reduced when an additional word was interpolated between two associated words, a finding which was seen to support the location shift model rather than the spreading activation model. The idea of backward activation, however, raises the question whether the interference effect of the interpolated item might not be increased or reduced by manipulating the likelihood of backward third-tofirst-word activation.

A final note is in order. Clearly, we are not ready yet for a precise statement regarding the processes underlying the association effect in lexical decision. Yet the formulation presented above suggests several hypotheses. First, if indeed the backward activation process is automatic and the forward is attentional, we should expect inhibitory effects of context to be confined to forward effects only. Second, if backward processes are automatic, they should continue to operate regardless of the level of attentional processes (in contrast to what is seen in Figure 2). Third, if under backward conditions the prime must be affecting the processing that takes place after the initial recognition of the test word, the interaction between context and visual quality of the stimulus should be weaker under these conditions than under forward conditions. Fourth, we should expect the magnitude of backward context effects to be unaffected by the validity with which the prime predicts the target 
word; forward context effects, in contrast, should be confined to high-validity conditions. Fifth, the word relations that are critical in determining magnitude of context effects may be expected to differ in significant ways for backward and forward activation. These and other hypotheses must be examined before a precise model can be worked out.

\section{REFERENCE NOTES}

1. Hekannen, S. Word recognition and the lexical decision task. Unpublished master's thesis, University of South Florida, 1977.

2. Killion, T. H. Activation of multiple meanings in the processing of lexical ambiguity. Unpublished doctoral dissertation, University of Oregon, 1978.

3. Swinney, D. Does context direct lexical access? Paper presented at the meeting of the Midwestern Psychological Association, Chicago, May 1976.

\section{REFERENCES}

AJzen, I. Intuitive theories of events and the effects of base-rate information on predictions. Journal of Personality and Social Psychology, 1977, 35, 303-314.

Barber, P., \& Rushton, J. P. Experimenter bias and subliminal perception. British Journal of Psychology, 1975, 66, 357-372.

BECKER, C. A. Semantic context effects in visual word recognition: An analysis of semantic strategies. Memory \& Cognition, $1980,8,493-512$.

Clark, H. H. The language-as-fixed-effect fallacy: A critique of language statistics in psychological research. Journal of Verbal Learning and Verbal Behavior, 1973, 12, 335-359.

Collins, A. M., \& LofTus, E. F. A spreading-activation theory of semantic processing. Psychological Review, 1975, 82, 407-428.

Fischlen, I. Associative facilitation without expectancy in a lexical decision task. Journal of Experimental Psychology: Human Perception and Performance, 1977, 3, 18-26. (a)

Fischler, I. Semantic facilitation without association in a lexical decision task. Memory \& Cognition, 1977, 5, 335-339. (b)

Fischler, I.. \& Goodman, G. O. Latency of associative activation in memory. Journal of Experimental Psychology: Human Perception and Performance, 1978, 4, 455-470.

JACOBson, J. Z. Effects of association upon masking and reading latency. Canadian Journal of Psychology, 1973, 27, 58-69.

Jenkins, P. M., \& Cofer, C. N. An exploratory study of discrete free association in compound verbal stimuli. Psychological Reports, 1957, 3, 599-602.

KePPEL, G., \& Strand, B. Z. Free association responses to the primary responses and other responses selected from the PalermoJenkins norms. In L. Postman \& G. Keppel (Eds.), Norms of word association. New York: Academic Press, 1970.

Kučera, H., \& Francis, W. N. Computational analysis of present day American English. Providence: Brown University Press, 1967.

Lortus, E. F. Category dominance, instance dominance, and categorization time. Journal of Experimental Psychology, 1973, 97, 70-74.

LoFTus, E. F. Spreading activation within semantic categories: Comments on Rosch's cognitive representations of semantic categories. Journal of Experimental Psychology: General, 1975, 104, 234-240.

Massaro, D. W., Jones, R. D., Lipscoma, C., \& Scholz, R. Role of prior knowledge on naming and lexical decisions with good and poor stimulus information. Journal of Experimental Psychology: Human Learning and Memory, 1978, 4, 498-512.

Meyer, D. E., \& Schvaneveldt, R. W. Facilitation in recogniz- ing pairs of words: Evidence of a dependence between retrieval operations. Journal of Experimental Psychology, 1971, 90, 227-243.

Moran, L. J. Generality of word-association response sets. Psychological Monograph, 1966, 80(4, Whole No. 612).

NEELY, J. H. Semantic priming and retrieval from lexical memory: Evidence for facilitatory and inhibitory processes. Memory \& Cognition, 1976, 4, 648-654.

Palermo, D. S., \& Jenkins, J. J. Word association norms: Grade school through college. Minneapolis: University of Minnesota Press, 1964.

Posner, M. I., \& SNyder, C. R. R. Attention and cognitive control. In R. L. Solso (Ed.), Information Processing and Cognition. Hillsdale, N.J: Erlbaum, 1975. (a)

Posner, M. I., \& SNyder, C. R. R. Facilitation and inhibition in the processing of signals. In P. M. A. Rabbitt (Ed.), Attention and performance $V$. London: Academic Press, 1975. (b)

Razran, G. Semantic and phonetographic generalizations of salivary conditioning to verbal stimuli. Journal of Experimental Psychology, 1949, 39, 642-652.

Rosch, E. Cognitive representations of semantic categories. Journal of Experimenial Psychology: General, 1975, 104, 192233. (a)

Rosch, E. Reply to Loftus. Journal of Experimental Psychology: General, 1975, 104, 241-243. (b)

Schmid, R. On the spread of semantic excitation. Psychological Research, 1976, 38, 333-353. (a)

Schmidt, R. Semantic expectancy effects on word access. Psychological Research, 1976, 39, 147-161. (b)

Schneider, W., \& Shiffrin, R. M. Controlled and automatic human information processing: I. Detection search and attention. Psychological Review, 1977, 84, 1-66.

Schubert, R. E., \& Eimas, P. D. Effects of context on the classification of words and nonwords. Journal of Experimental Psychology: Human Perception and Performance, 1977, 3, 27-36.

Schvaneveldt, R. W., \& Meyer, D. E. Retrieval and comparison processes in semantic memory. In S. Kornblum (Ed.), Attention and performance IV. New York: Academic Press, 1973.

Schvaneveldt, R. W., Meyer, D. E., \& Becker, C. A. Lexical ambiguity, semantic context, and visual recognition. Journal of Experimental Psychology: Human Perception and Performance, 1976, 2, 243-256.

Shiffrin, R. M., \& Schneider, W. Controlled and automatic human information processing: II. Perceptual learning, automatic attending, and a general theory. Psychological Review, 1977, 84, 127-190.

Spence, D. P., \& Holland, B. The restricting effects of awareness. Journal of Abnormal and Social Psychology, 1962, 64, 163-174.

SpEnCE, D. P., \& Sмiтh, G. J. W. Experimenter bias against subliminal perception? Comments on a replication. British Journat of Psychology, 1977, 68, 279-280.

Tweedy, J. R., Lapinski, R. H., \& Schvaneveldi, R. W. Semantic context effects on word recognition: Influence of varying the proportion of items presented in an appropriate context. Memory \& Cognition, 1977, 5, 84-89.

UNDERWOOD, B. J. False recognition produced by implicit verbal responses. Journal of Experimental Psychology, 1965, 70, 122129.

WARREN, R. E. Stimulus encoding and memory. Journal of Experimental Psychology, 1972, 94, 90-100.

WARREN, R. E. Association, directionality, and stimulus encoding. Journal of Experimental Psychology, 1974, 102, 151-158.

(Received for publication August 19, 1980; revision accepted March S, 1981.) 